\title{
Analysis of the interaction between personality dysfunction and traits in the statistical prediction of physical aggression: Results from outpatient and community samples
}

\author{
Philippe Leclerc ${ }^{1}$ (i) | Claudia Savard ${ }^{2}$ (i) | David D. Vachon ${ }^{3}$ | \\ Jonathan Faucher $^{1}$ | Maude Payant ${ }^{4}$ | Mireille Lampron ${ }^{5}$ | Marc Tremblay ${ }^{6}$ | \\ Dominick Gamache ${ }^{1}$ (i)
}

${ }^{1}$ Département de psychologie, Université du Québec à Trois-Rivières,

Trois-Rivières, Québec, Canada

${ }^{2}$ Département des fondements et pratiques en éducation, Université Laval, Québec City, Québec, Canada

${ }^{3}$ Department of Psychology, McGill University, Montréal, Québec, Canada

${ }^{4}$ Département de psychologie, Université du Québec à Montréal, Montréal, Québec, Canada

${ }^{5}$ École de psychologie, Université Laval, Québec City, Québec, Canada

${ }^{6}$ Centre intégré universitaire de santé et de services sociaux de la CapitaleNationale, Québec City, Québec, Canada

\section{Correspondence}

Philippe Leclerc, Département de psychologie, Université du Québec à Trois-Rivières, 3600 Sainte-Marguerite (Pavillon Michel-Sarrazin), Trois-Rivières, QC, G9A 5H7, Canada.

Email: philippe.leclerc@uqtr.ca

\section{Funding information}

Centre intégré universitaire de santé et de services sociaux de la Capitale-Nationale, Grant/Award Number: None; Social Sciences and Humanities Research Council of Canada, Grant/Award Number: 892-2017-3003

\begin{abstract}
The Alternative Model for Personality Disorders (AMPD), included in the Diagnostic and Statistical Manual of Mental Disorders (5th ed.) and the World Health Organization's International Classification of Diseases (11th ed.; ICD-11) are, respectively, hybrid categorical-dimensional and dimensional frameworks for personality disorders (PDs). Both models emphasize personality dysfunction and personality traits. Previous studies investigating the links between the AMPD and ICD-11, and self-reported physical aggression have mostly focused on traits and did not take into account the potential interaction between personality dysfunction and traits. Thus, the aim of this study is to identify dysfunction*trait interactions using regression-based analysis. Outpatients with personality disorder from a specialized public clinic ( $N=285)$ and community participants $(N=995)$ were recruited to complete self-report questionnaires. Some small-size, albeit significant and clinically/ conceptually meaningful personality dysfunction*trait interactions were found to predict physical aggression in both samples. Interaction analyses might further inform, to some degree, about the current discussion pertaining to the potential redundancy between dysfunction and traits, the optimal personality dysfunction structure (in the case of the AMPD), as well as clinical assessment based on AMPD/ICD-11 PD frameworks.
\end{abstract}

\section{INTRODUCTION}

The Alternative Model for Personality Disorders (AMPD), included in Section III of the Diagnostic and
Statistical Manual of Mental Disorders (5th ed. DSM-5) (American Psychiatric Association, 2013) and the World Health Organization's International Classification of Diseases (11th ed.; ICD-11) (Bach \& First, 2018) are, 
respectively, hybrid categorical-dimensional and dimensional frameworks for personality disorders (PDs). Both seek to alleviate many of the shortcomings of the categorical classification (see Hopwood et al., 2018) and position personality dysfunction at their core, which is characterized by self and interpersonal pathology (referred to as "Criterion A", in the case of the DSM-5). For AMPD Criterion A, self and interpersonal pathology is more specifically defined by Identity and Self-Direction disturbances, and Empathy and Intimacy disturbances, respectively. In both models, personality dysfunction is theoretically meant to reflect personality-specific impairments (i.e., not present in "syndromic" disorders) and to help at ruling whether a person has a PD diagnosis or not. Both the DSM-5 and the ICD-11 are also composed of pathological personality traits, allowing a fine-grained, individual profile (referred to as "Criterion B" in DSM-5's AMPD, and as "Trait domain qualifiers" in the ICD-11): Negative Affectivity, Detachment, Antagonism/Dissociality, Disinhibition, Psychoticism (AMPD only), and Anankastia (ICD-11 only). ${ }^{1}$

In the ICD-11, personality dysfunction is conceptualized as a single, global indicator, and it does not have an official instrument (Bach \& Simonsen, 2021). The AMPD Criterion A can be assessed with the Level of Personality Functioning Scale (a clinician-rated grid), but it does not have an official self-reported instrument. Its conceptualization is also less consensual. Indeed, the DSM-5 itself is ambiguous as to how the Criterion A structure should be viewed (i.e., unidimensional versus multidimensional) (Sleep et al., 2019); as a result, multiple instruments based on different plausible structures for Criterion A are currently available (Gamache et al., 2019; Morey, 2017; Siefert et al., 2020; Weekers et al., 2019). Morey (2017, 2019), who was part of the Personality and Personality Disorders Work Group that developed Criterion A, argues that its structure is best represented by a single score, which was recently supported by bifactor analyses that corroborated a unidimensional spectrum of personality dysfunction (Hummelen et al., 2020; Zimmermann et al., 2020). In the broader AMPD research community, academics are more divided, and it was suggested that Criterion A could be best represented by two dimensions or four distinct elements (Gamache et al., 2019; Siefert et al., 2020; Weekers et al., 2019). An intermediate position, in which both the total score and its four elements have merits from psychometric and clinical standpoints, has also been advocated (Buer Christensen et al., 2020; Gamache et al., 2019; Siefert et al., 2020).

Previous research using simultaneously personality dysfunction and traits aimed mainly at identifying their mutual incremental validity. In the context of the AMPD, when they are "pitted one against another" (e.g., through hierarchical regression analysis) or jointly factor analyzed, their respective addition or delineation is not so obvious, and Criterion B seems to explain more variance than Criterion A in general (at least, using self-reports) (Zimmermann et al., 2019). Different explanations have been put forth, for example, that dysfunction and traits refer more or less to the same constructs but are rooted in different theoretical paradigms (Zimmermann et al., 2020). However, the interaction between dysfunction and traits has been understudied thus far, which has been underscored for both the AMPD (Meehan et al., 2019) and the ICD-11 (Bach \& Simonsen, 2021). Indeed, if redundancy is complete, then no interaction should be obtained (e.g., through regressions or factor analyses incorporating an interaction term) (Sexton et al., 2019). This is a promising avenue to document both the combined utility of dysfunction and traits, as well as what is the most appropriate personality dysfunction structure (in the case of the AMPD). For instance, some Criterion A elements (Self-Direction, Empathy) and traits (Detachment, Antagonism) have shown interactions in the prediction of self-reported satisfaction in work and love relations (Sexton et al., 2019). In another study, the Severity Indices of Personality Problems (SIPP-118) (Verheul et al., 2008) dimensions (a measure of personality dysfunction) also moderated the relationship between three Criterion B traits (Negative Affectivity, Detachment, Psychoticism) and psychological distress (Benzi et al., 2019). Those studies supported both a non-static level of analysis (for the AMPD and, by extension, the ICD-11), as well as the contention that separate Criterion A elements (in the case of the AMPD) might be relevant.

One application that could benefit from a dysfunction*trait analysis is physical aggression. Both the psychodynamic formulations pertaining to personality dysfunction (e.g., Kernberg's Level of Personality Organization) (Caligor et al., 2018) and the multivariate paradigm pertaining to personality traits (e.g., Hyatt et al., 2018) consider personality as an important predictor for the perpetration of aggression. Nevertheless, both traditions have been historically quite isolated, leading to different lines of research, a gap mostly perpetuated in the existing AMPD/ICD-11 literature pertaining to aggression. Specifically, it was reported that the Criterion A total score predicted different types of intimate partner violence (IPV) (Gamache, Savard, Lemieux, et al., 2021). Other studies identified how personality traits predict aggression (Dunne et al., 2018; Dunne et al., 2021; Sleep et al., 2018) and IPV (Dowgwillo et al., 2016; Munro \& Sellbom, 2020). However, to the best of our knowledge, no published study has focused on the interplay between personality dysfunction and traits in the statistical 
prediction of physical aggression, that is, whether the two might interact. This issue is particularly important since the level of personality dysfunction (akin to Kernberg's severity axis in his model) might be an important moderator of the effect of traits on aggression; a better understanding of the issue would have direct clinical implications (e.g., in the assessment of a patient's propensity toward aggression)—for instance, a patient with high personality impairment and an elevation of some traits (e.g., Antagonism/Dissociality) could have a higher propensity toward aggression than another patient with low personality impairment (other things being equal).

\section{The present study}

This article aims at identifying how personality dysfunction and traits interact in their relation to self-reported physical aggression in order to gain a more nuanced conceptual understanding of the AMPD/ICD-11 models and to inform assessment. Since the discussion pertaining to the Criterion A structure is not resolved (in the case of the AMPD), different conceptualizations will be investigated. When personality dysfunction is conceptualized as a single-factor structure (as in the ICD-11 and as proposed for the AMPD by, for example, Morey, 2017, 2019), it is expected that it will statistically predict aggression, based on previous results (Gamache, Savard, Lemieux, et al., 2021). When it is conceptualized as a four-factor structure (as proposed by other AMPD researchers) (Buer Christensen et al., 2020; Gamache et al., 2019; Siefert et al., 2020), it is expected that only Empathy (impairment) will statistically predict aggression, based on previous results (Gamache et al., 2019). With regard to personality traits, it is not clear which domains will predict aggression, as some inconsistencies exist in the literature. Dunne et al. (2018) found no significant trait predictors, but their group subsequently reported (Dunne et al., 2021) that AMPD Antagonism, Disinhibition, and Negative Affectivity (low) were predictive of aggression, using a shorter Criterion B instrument. Sleep et al. (2018) reported the exact same significant predictors, except for AMPD Negative Affectivity (high). In addition to AMPD Antagonism and Disinhibition, Dowgwillo et al. (2016) reported that Detachment significantly predicted IPV. Therefore, the following hypothesis must be considered with caution: Antagonism and Disinhibition will most likely statistically predict aggression, while prediction by Negative Affectivity and Detachment is possible although uncertain. Personality dysfunction*trait interactions have been reported only in non-aggression studies (Benzi et al., 2019; Sexton et al., 2019). Nevertheless, the following hypothesis can be formulated: dysfunction will moderate traits (i.e., higher personality impairment will increase the association between statistically significant trait predictors and aggression), in line with Kernberg's model.

\section{METHODS}

\section{Participants and procedures}

Two samples of mainly French-speaking Canadians from the Province of Quebec, Canada were recruited in this study. The first sample is composed of PD outpatients ( $N=285,177$ females, 108 males, $M_{\text {age }}=33.72$ years old, $S D=10.59,18-69$ range) recruited from a public clinic specialized in the assessment and treatment of severe PDs in Quebec City, Canada, between September 2017 and February 2020. Only a minority had completed a university degree (19\%), was working full- or part-time (48\%), and was in a romantic relationship or married (37\%). Data were collected during the computer-based intake protocol at the clinic prior to a clinical interview and participants were presented with the possibility to give access to their data for research purposes. Access to services is available for adult patients ( $\geq 18$ years old) from the Quebec City area upon referral from a general physician or psychiatrist, and requires at least one formal DSM-5 Section II PD diagnosis. Diagnoses from the referring physician or psychiatrist are confirmed during an intake interview by a clinical psychologist, and all diagnoses are then reviewed by a team of six clinical psychologists during a weekly meeting. PD diagnosis based on DSM-5 Section II was available for 254 patients (87.9\%): 65 narcissistic (22.5\%), 48 borderline-narcissistic (16.6\%), 46 borderline (15.9\%), 41 mixed (i.e., $\geq 3$ PDs; $14.2 \%), 23$ not otherwise specified (8.0\%), 10 schizotypal (3.5\%), six schizoid (2.1\%), five syndromic (i.e., non-PD main diagnosis; $1.7 \%$ ), four antisocial (1.4\%), four histrionic (1.4\%), and two paranoid (0.7\%). The second sample is comprised of community participants $(N=995$, 764 females, 219 males, six of other gender identity, six who did not answer, $M_{\text {age }}=46.16$ years old, $S D=13.69$, 18-84 range), who were recruited online from September 2020 to February 2021 by snowball sampling and through mailing lists from a psychology organization and Facebook advertisement. A majority had completed a university degree (54\%), was working full- or part-time (59\%), and was in a romantic relationship or married (64\%). All data were inspected for valid responding (e.g., absence of repeated patterns of 1-2-3), missing data, and conformity with multivariate analysis assumptions. Then, a Mahalanobis Distance Analysis was performed 
with all predictors to identify multivariate outliers $(p<0.001)$, which have already been excluded.

\section{Measures}

\section{Personality dysfunction}

The Self and Interpersonal Functioning Scale (SIFS) (Gamache et al., 2019) is a 24-item measure that assesses personality dysfunction based on the AMPD Criterion A. It has also demonstrated its validity to determine severity degrees of personality pathology based on the ICD-11 (Gamache, Savard, Leclerc, et al., 2021). It was originally developed for screening purposes based on a multi-element Criterion A conceptualization (i.e., four elements-Identity, Self-direction, Empathy, and Intimacy—organized into a higher-order personality dysfunction factor). An independent content comparative analysis (Waugh et al., 2021) showed that the SIFS has good content validity. Its factor structure makes it also an appropriate choice to investigate both a one- and a fourfactor Criterion A conceptualization. The outpatients were administered the original SIFS (four elements $[\alpha=0.66-0.72]$ and global score $[\alpha=0.84])$, while the community participants were administered a slightly revised version which covers the exact same subscales but with slightly less items (20) and using no reversed wording items (four elements $[\alpha=0.67-0.88]$ and global score $[\alpha=0.90])$. Items are rated on a five-point Likert scale in both versions.

\section{Personality traits}

The Personality Inventory for DSM-5 Faceted Brief Form (PID-5-FBF) (Maples et al., 2015) is an abbreviated 100-item version of the original 220-item PID-5 (Krueger et al., 2012) (French validation by Roskam et al., 2015) measuring the AMPD Criterion B. It is comprised of 25 maladaptive personality facets organized into five higher-order personality domains: Negative Affectivity, Detachment, Antagonism, Disinhibition, and Psychoticism ( $\alpha=0.82-0.92$ [outpatients] and 0.79-0.90 [community]). In addition to being the official Criterion $B$ measure for the AMPD, it can be considered as a legitimate (yet imperfect; e.g., Anankastia is not fully covered) proxy for ICD-11 traits (McCabe \& Widiger, 2020). Only the higher-order traits were used. All analyses were computed using both APA's three facets procedure (American Psychiatric Association, 2013) and Krueger et al.'s algorithms (Krueger et al., 2012) in the coding of traits to widen the scope of our conclusions (see Watters et al., 2019). Indeed, some seemingly important facet predictors based on previous results (Dunne et al., 2018) are not incorporated in the APA scoring procedure (e.g., Hostility and Risk Taking). Items are rated on a four-point Likert scale.

\section{Physical aggression}

The Short-Form Buss-Perry Aggression Questionnaire (BPAQ-SF; Bryant \& Smith, 2001; French validation by Genoud \& Zimmermann, 2009) is an abbreviated 12-item and psychometrically improved measure of the 29-item BPAQ (Buss \& Perry, 1992) assessing physical and verbal aggression, anger, and hostility. Only the Physical Aggression subscale ( $\alpha=0.86$ [outpatients] and 0.61 [community]) was used to focus on the physical component of aggression, as well as to avoid item content overlap with the SIFS or the PID-5-FBF (e.g., PID-5-FBF's Hostility "I have a very short temper" resembles BPAQSF's Anger "I have trouble controlling my temper"). Items are rated on a six-point Likert scale.

\section{Analytic strategy}

Analyses were conducted using IBM SPSS 27. First, descriptive statistics and bivariate correlational analyses (between personality functioning, traits, and physical aggression) were computed. Second, two multiple regression models were computed including either the SIFS total score or all four elements, as well as traits to identify main effects. Since the number of predictors is uneven when using the total score or the four elements, the Prediction Sum-of-Squares (PRESS) statistic (D. M. Allen, 1974) was computed manually to derive the Predicted $R^{2}$, which is unaffected by the number of predictors entered. This indicator also informs on the model's capacity to provide accurate predictions by sequentially taking out each case of the model and then using the predicted value to provide a new estimation. Considering that a regressor might obtain a nonzero regression weight even if it is uncorrelated with the dependent variable (Darlington \& Hayes, 2016), all SIFS and PID-5-FBF variables were retained in the regression analyses. Third, to identify interactions, a series of 25 hierarchical multiple regression analyses were computed including a PID-5-FBF trait and a SIFS element (4 elements $* 5$ traits $=20$ models) or the total score ( 1 total dysfunction score*5 traits $=5$ models) at Step 1, and an interaction term (i.e., element*trait) at Step 2. All predictors were mean-centered prior to computing products of variables to reduce microcolinearity (Iacobucci 

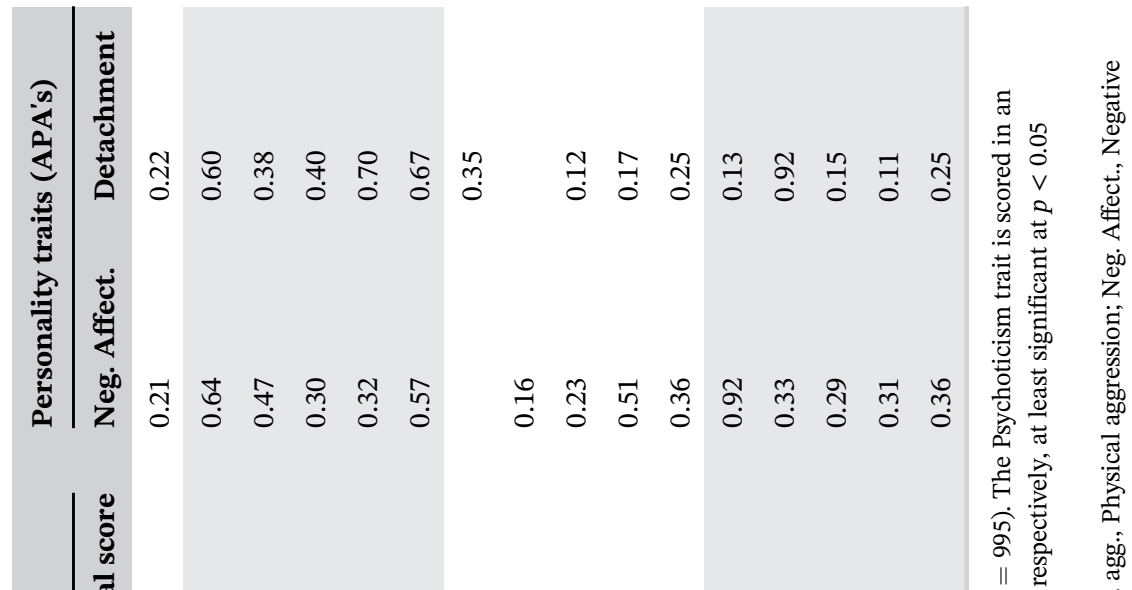

?

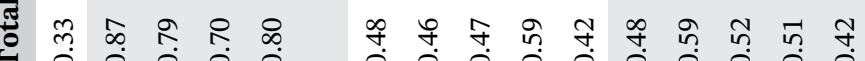

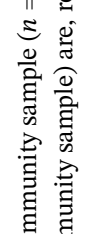

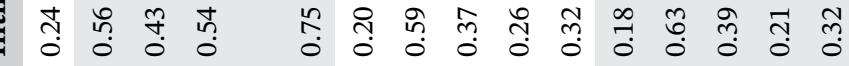

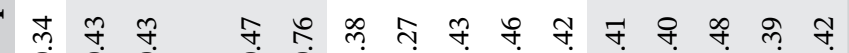

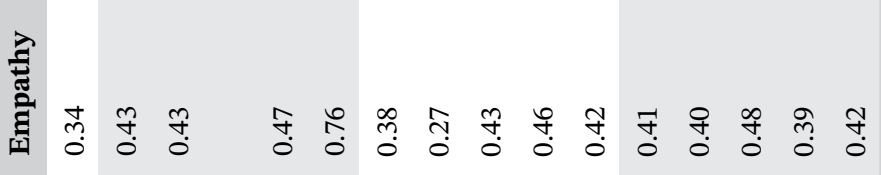

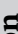

(1)

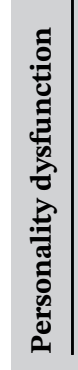

荘

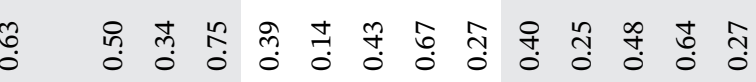

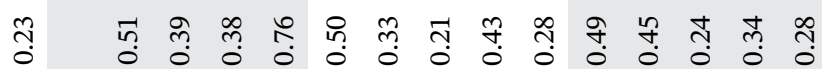

ד.

永

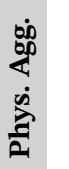

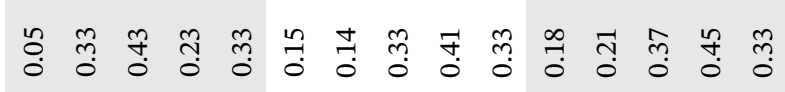

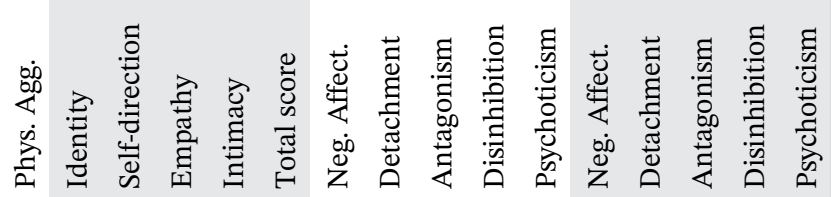




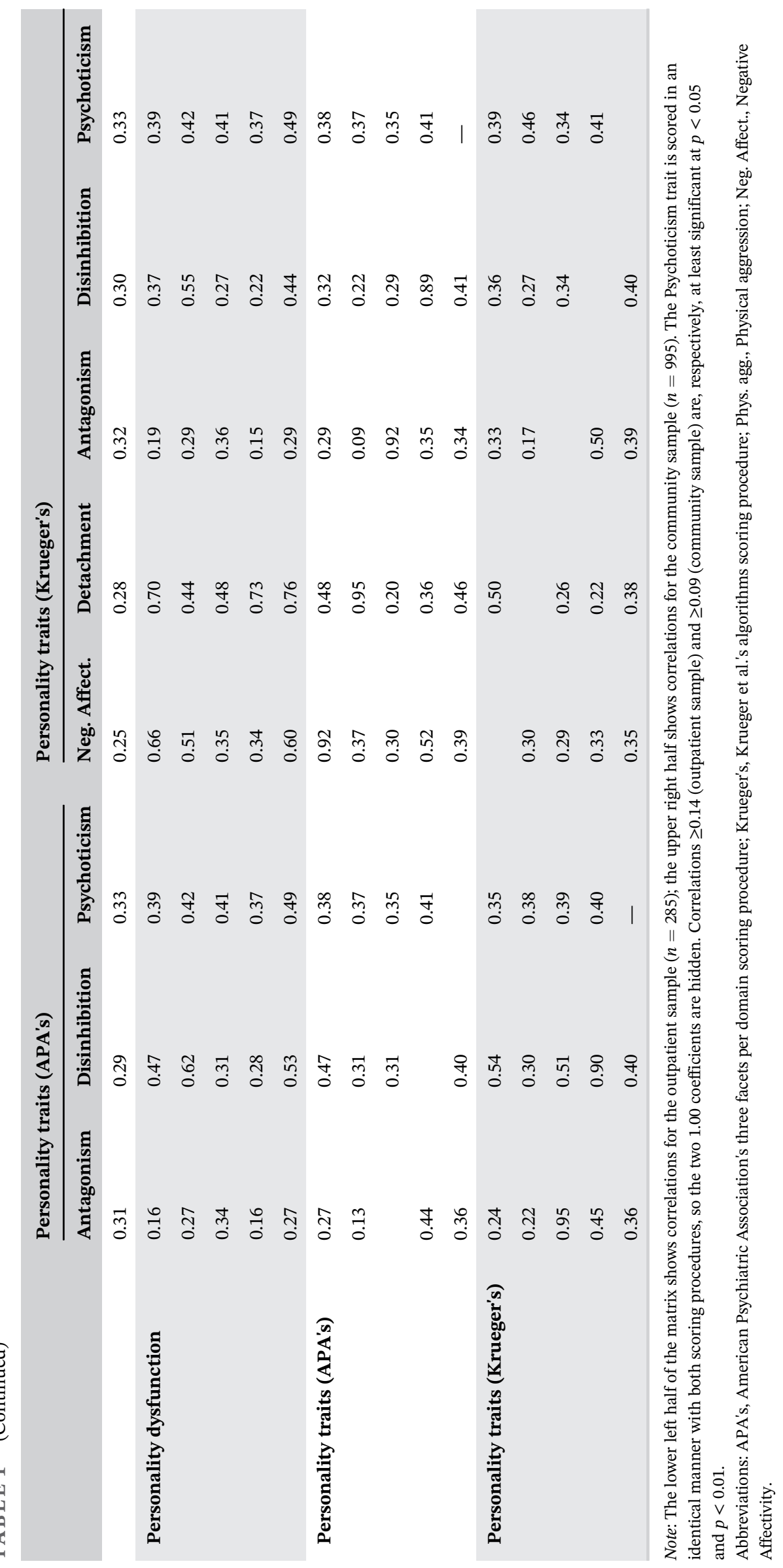


et al., 2016). All analyses were computed with both scoring procedures $(2$ procedures $* 25$ models $=50$ models) for each sample. Balancing statistical power and significance level ( $\alpha$ level) is a complicated matter when probing interactions, since much more power is needed. Indeed, most researchers seek at least $80 \%$ power to identify main effects-in practice, to detect an interaction effect half that size, power will actually be around $10 \%$ (Durand, 2013). Indeed, the required sample size to probe interactions grows dramatically for a decreasing interaction size. For instance, to detect an interaction effect that is half the size of the main effect, 16 times more power would theoretically be necessary (Durand, 2013). While elevating the $\alpha$ level is generally not advisable (e.g., to put it at $\alpha=0.10$ ) (Durand, 2013), a Bonferroni correction is usually too conservative for multiple regression analyses and appropriate only to identify a parsimonious set of predictors in a model (Mundfrom et al., 2006), which is not the objective of the present study. Therefore, the $\alpha$ level will be kept at 0.05 . To guard against Type I inflation, a particular emphasis will be put on replication between samples and theoretical soundness.

\section{RESULTS}

Descriptive statistics and reliability indices are provided as supplemental material (see Tables S1 and S2).
Bivariate zero-order Pearson correlations among physical aggression, personality dysfunction, and traits (for both samples) are provided in Table 1. Significant correlations with aggression were as follows: all traits (using both scoring procedures), and all personality dysfunction elements (except Identity in outpatients) in addition to the global score. For the main effect analysis, the complete outputs are shown in Tables 2 and 3 (traits using APA's procedure and Krueger et al.'s algorithms, and personality dysfunction either as a single score or as four elements). The two scoring procedures globally yielded similar results, that is, Identity (outpatients) and Empathy (community and outpatients) were significant personality dysfunction predictors of aggression in both cases, but some differences for the significant trait predictors were found. When personality dysfunction was represented by a single score, it was a statistically significant predictor for the community sample only.

For the interaction analyses, a synthesis of all interaction models is provided in Tables 4 and 5 (only the output at Step 2 is shown, i.e., the interaction terms). Some statistically significant dysfunction*trait interactions were found in both samples: for the outpatient sample: Empathy*Disinhibition (APA's: $\beta=0.12, \Delta R^{2}=1.4 \%$; Krueger's: $\beta=0.14, \Delta R^{2}=1.9 \%$ ), Intimacy*Antagonism (APA's: $\beta=-0.13, \Delta R^{2}=1.4 \%$; Krueger's: $\beta=-0.14$, $\Delta R^{2}=1.7 \%$ ), Total score*Disinhibition (APA's: $\beta=0.13$, $\Delta R^{2}=1.7 \%$; Krueger's: $\beta=0.11, \Delta R^{2}=1.1 \%$; for the

TA B LE 2 Multiple regression analysis of personality dysfunction and traits to statistically predict physical aggression—Outpatient with personality disorder sample $(N=285)$

\begin{tabular}{|c|c|c|c|c|c|c|c|c|c|}
\hline & Predictors & \multicolumn{2}{|l|}{ APA's } & \multicolumn{2}{|c|}{ Krueger's } & \multicolumn{2}{|l|}{ APA's } & \multicolumn{2}{|c|}{ Krueger's } \\
\hline \multirow[t]{3}{*}{ Personality dysfunction } & Identity & -0.26 & $<0.001$ & -0.27 & $<0.001$ & & & & \\
\hline & Self-direction & 0.09 & 0.248 & 0.04 & 0.642 & & & & \\
\hline & Empathy & 0.28 & $<0.001$ & 0.28 & $<0.001$ & & & & \\
\hline \multirow[t]{6}{*}{ Personality traits } & Negative affectivity & -0.08 & 0.218 & 0.00 & 0.950 & -0.14 & 0.034 & -0.05 & 0.450 \\
\hline & Detachment & 0.05 & 0.471 & 0.08 & 0.272 & 0.01 & 0.838 & 0.05 & 0.474 \\
\hline & Antagonism & 0.05 & 0.410 & 0.06 & 0.352 & 0.12 & 0.057 & 0.15 & 0.022 \\
\hline & Disinhibition & 0.28 & $<0.001$ & 0.33 & $<0.001$ & 0.31 & $<0.001$ & 0.32 & $<0.001$ \\
\hline & Predicted $R^{2}$ & 0.26 & & 0.29 & & 0.19 & & 0.22 & \\
\hline & $\Delta F$ & 14.02 & $<0.001$ & 15.80 & $<0.001$ & 13.95 & $<0.001$ & 15.89 & $<0.001$ \\
\hline
\end{tabular}

Note: Statistically significant predictors are italicized and bolded. For all variables, higher scores mean higher impairment/pathology/aggression. Dependent variable: Physical Aggression subscale from the Short-Form Buss-Perry Aggression Questionnaire.

Abbreviations: APA's, American Psychiatric Association's three facets per domain scoring procedure; Krueger's, Krueger et al.'s algorithms scoring procedure. 
T A B L E 3 Multiple regression analysis of personality dysfunction and traits to statistically predict physical aggression—Community sample $(N=995)$

\begin{tabular}{|c|c|c|c|c|c|c|c|c|c|}
\hline & Predictors & \multicolumn{2}{|l|}{ APA's } & \multicolumn{2}{|c|}{ Krueger's } & \multicolumn{2}{|l|}{ APA's } & \multicolumn{2}{|c|}{ Krueger's } \\
\hline \multirow[t]{3}{*}{ Personality dysfunction } & Identity & -0.03 & 0.569 & -0.09 & 0.095 & & & & \\
\hline & Self-direction & 0.05 & 0.277 & 0.05 & 0.257 & & & & \\
\hline & Empathy & 0.16 & $<0.001$ & 0.15 & $<0.001$ & & & & \\
\hline \multirow[t]{6}{*}{ Personality traits } & Negative affectivity & -0.01 & 0.908 & 0.03 & 0.534 & -0.04 & 0.230 & -0.01 & 0.698 \\
\hline & Detachment & 0.05 & 0.261 & 0.13 & 0.009 & 0.02 & 0.678 & 0.09 & 0.058 \\
\hline & Antagonism & 0.16 & $<0.001$ & 0.16 & $<0.001$ & 0.19 & $<0.001$ & 0.20 & $<0.001$ \\
\hline & Disinhibition & 0.10 & 0.009 & 0.12 & 0.001 & 0.09 & 0.009 & 0.11 & 0.001 \\
\hline & Predicted $R^{2}$ & 0.17 & & 0.18 & & 0.18 & & 0.19 & \\
\hline & $\Delta F$ & 26.79 & $<0.001$ & 28.43 & $<0.001$ & 37.37 & $<0.001$ & 39.05 & $<0.001$ \\
\hline
\end{tabular}

Note: Statistically significant predictors are italicized and bolded. For all variables, higher scores mean higher impairment/pathology/aggression. Dependent variable: Physical Aggression subscale from the Short-Form Buss-Perry Aggression Questionnaire.

Abbreviations: APA's, American Psychiatric Association's three facets per domain scoring procedure; Krueger's, Krueger et al.'s algorithms scoring procedure.

T A B L E 4 Regression-based analysis of interaction between personality dysfunction and traits to statistically predict physical aggression: A synthesis of possible models-Outpatient with personality disorder sample $(N=285)$

Personality dysfunction

\begin{tabular}{|c|c|c|c|c|c|c|c|c|c|c|c|c|c|c|}
\hline \multicolumn{3}{|c|}{ Identity } & \multicolumn{3}{|c|}{ Self-direction } & \multicolumn{3}{|c|}{ Empathy } & \multicolumn{3}{|c|}{ Intimacy } & \multicolumn{3}{|c|}{ Total score } \\
\hline$\Delta \boldsymbol{F}$ & $p$ & $\Delta R^{2}$ & $\Delta \boldsymbol{F}$ & $p$ & $\Delta R^{2}$ & $\Delta \boldsymbol{F}$ & $p$ & $\Delta R^{2}$ & $\Delta \boldsymbol{F}$ & $p$ & $\Delta R^{2}$ & $\Delta F$ & $p$ & $\Delta R^{2}$ \\
\hline
\end{tabular}

\section{Personality traits (APA's)}

$\begin{array}{llllllllllllllll}\text { Neg. Affect. } & 0.05 & 0.829 & 0.000 & 0.44 & 0.507 & 0.001 & 0.11 & 0.742 & 0.000 & 1.05 & 0.306 & 0.003 & 2.91 & 0.089 & 0.009 \\ \text { Detachment } & 0.72 & 0.396 & 0.003 & 0.01 & 0.933 & 0.000 & 3.47 & 0.063 & 0.010 & 0.84 & 0.361 & 0.003 & 1.03 & 0.311 & 0.003 \\ \text { Antagonism } & 1.24 & 0.267 & 0.004 & 1.90 & 0.169 & 0.006 & 0.29 & 0.594 & 0.001 & \mathbf{4 . 5 7} & 0.034 & 0.014 & 1.85 & 0.174 & 0.006 \\ \text { Disinhibition } & 0.77 & 0.380 & 0.002 & 1.16 & 0.283 & 0.003 & \mathbf{5 . 4 2} & 0.021 & 0.014 & 3.09 & 0.080 & 0.009 & \mathbf{6 . 0 2} & 0.015 & 0.017 \\ \text { Psychoticism } & 1.42 & 0.235 & 0.004 & 0.18 & 0.674 & 0.001 & 0.07 & 0.789 & 0.000 & 0.02 & 0.885 & 0.000 & 0.01 & 0.904 & 0.000\end{array}$

Personality traits (Krueger's)

\begin{tabular}{|c|c|c|c|c|c|c|c|c|c|c|c|c|c|c|c|}
\hline $\begin{array}{l}\text { Neg. } \\
\text { Affect. }\end{array}$ & 0.04 & 0.841 & 0.000 & 0.07 & 0.798 & 0.000 & 0.01 & 0.907 & 0.000 & 1.47 & 0.227 & 0.005 & 2.51 & 0.114 & 0.008 \\
\hline Detachment & 0.34 & 0.560 & 0.001 & 0.04 & 0.838 & 0.000 & 3.46 & 0.064 & 0.010 & 1.50 & 0.222 & 0.005 & 1.68 & 0.196 & 0.005 \\
\hline Antagonism & 0.27 & 0.603 & 0.001 & 0.67 & 0.414 & 0.002 & 0.02 & 0.879 & 0.000 & 5.67 & 0.018 & 0.017 & 0.98 & 0.323 & 0.003 \\
\hline Disinhibition & 0.02 & 0.892 & 0.000 & 0.15 & 0.697 & 0.000 & 7.74 & 0.006 & 0.019 & 2.97 & 0.086 & 0.008 & 3.97 & 0.047 & 0.011 \\
\hline Psychoticism & 1.42 & 0.235 & 0.004 & 0.18 & 0.674 & 0.001 & 0.07 & 0.789 & 0.000 & 0.02 & 0.885 & 0.000 & 0.01 & 0.904 & 0.000 \\
\hline
\end{tabular}

Note: Statistically significant $\Delta F$ are italicized and bolded. Dependent variable: Physical aggression. All models include a personality dysfunction element (e.g., Identity) or the total score and a trait (e.g., Negative Affectivity) at Step 1 (not presented) and an interaction term at Step 2 (e.g., Negative Affectivity*Identity). The numbers $\left(\Delta F\right.$ and $\Delta R^{2}$ ) represent the addition of Step 2 to Step 1, that is, the addition of the interaction term (e.g., Negative Affectivity*Identity). Abbreviations: APA's, American Psychiatric Association's three facets per domain scoring procedure; Krueger's, Krueger et al.'s algorithms scoring procedure; Neg. Affect., Negative Affectivity. 
TA B LE 5 Regression-based analysis of interaction between personality dysfunction and traits to statistically predict physical aggression: A synthesis of possible models-Community sample $(N=995)$

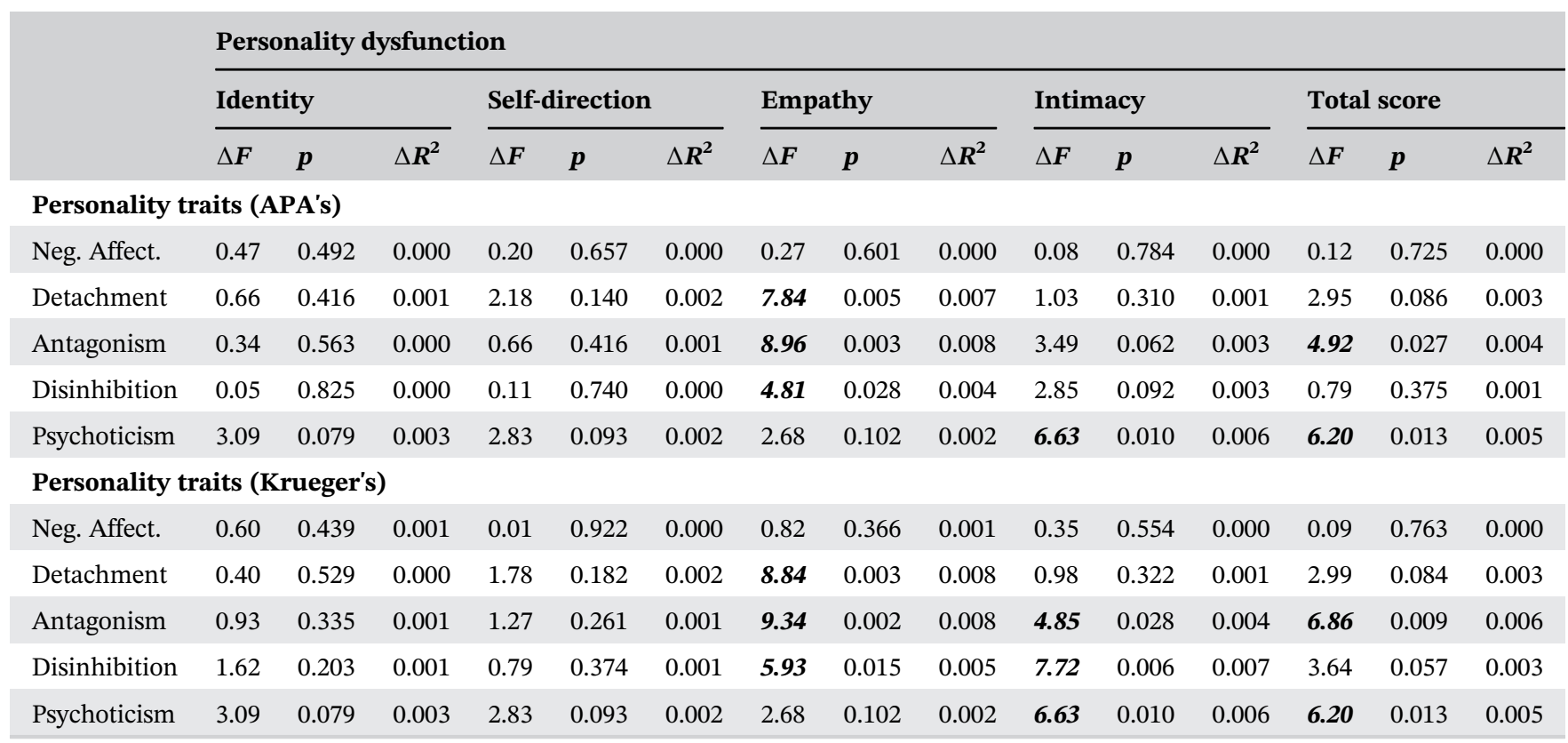

Note: Statistically significant $\Delta F$ are italicized and bolded. Dependent variable: Physical aggression (logarithmically transformed to improve univariate normality). All models include a personality dysfunction element (e.g., Identity) or the total score and a trait (e.g., Negative Affectivity) at Step 1 (not presented) and an interaction term at Step 2 (e.g., Negative Affectivity*Identity). The numbers $\left(\Delta F\right.$ and $\Delta R^{2}$ ) represent the addition of Step 2 to Step 1, that is, the addition of the interaction term (e.g., Negative Affectivity*Identity).

Abbreviations: APA's, American Psychiatric Association's three facets per domain scoring procedure; Krueger's, Krueger et al.'s algorithms scoring procedure; Neg. Affect., Negative Affectivity.

community sample: Empathy*Detachment (APA's: $\beta=0.09, \Delta R^{2}=0.7 \%$; Krueger's: $\beta=0.10, \Delta R^{2}=0.8 \%$, Empathy*Antagonism (APA's: $\beta=0.09, \quad \Delta R^{2}=0.8 \%$; Krueger's: $\beta=0.09, \Delta R^{2}=0.8 \%$ ), Empathy*Disinhibition (APA's: $\quad \beta=0.07, \quad \Delta R^{2}=0.4 \% ; \quad$ Krueger's: $\beta=.07$, $\Delta R^{2}=0.5 \%$ ), Intimacy*Antagonism (Krueger's: $\beta=0.07$, $\Delta R^{2}=0.4 \%$ ), Intimacy*Disinhibition (Krueger's: $\beta=0.08, \Delta R^{2}=0.7 \%$ ), Intimacy*Psychoticism (APA's: $\beta=0.08, \Delta R^{2}=0.6 \%$; Krueger's: $\beta=0.08, \Delta R^{2}=0.6 \%$ ), Total score*Antagonism (APA's: $\beta=0.07, \Delta R^{2}=0.4 \%$; Krueger's: $\left.\quad \beta=0.08, \quad \Delta R^{2}=0.6 \%\right), \quad$ Total score* $^{*}$ Psychoticism (APA's: $\beta=0.08, \Delta R^{2}=0.5 \%$; Krueger's: $\beta=0.08, \Delta R^{2}=0.5 \%$.

\section{DISCUSSION}

This study aimed at identifying dysfunction*trait interactions, based on the emerging AMPD/ICD-11 PD classifications, in the statistical prediction of aggression. The main hypothesis that higher personality dysfunction would moderate the association between significant traits and aggression was supported (in all but one model). A total of three small-size and conceptually meaningful interactions were found for the outpatient sample and eight for the community sample. Among those interactions, two were found in both samples.

This is the first study to report such effects in the AMPD/ICD-11 literature pertaining to aggression. All but one significant interaction effect had a positive regression coefficient, meaning that a PD outpatient or community person with both high levels of some pathological traits (Antagonism, Disinhibition, Psychoticism) and high personality impairment (Empathy, Intimacy, Total score) will have their aggression propensity increased by an interaction factor. Of note, results from both samples suggest that the interpersonal component of personality dysfunction (i.e., Empathy and Intimacy) is a more important moderator of aggression. Indeed, some interactions were significant in both samples (e.g., Empathy*Disinhibition, Intimacy*Antagonism). It is likely that social relationships are important "triggers" for the perpetration of aggression (e.g., having an argument with someone and feeling provoked). Intriguingly, the Antagonism*Intimacy interaction was positive for the community sample, but yielded a negative coefficient for the outpatient sample. It might be that the relation of Antagonism with physical aggression is more pronounced at low-moderate levels of Intimacy impairment (like what would be found in community participants or less impaired outpatients). Additionally, perhaps highly 
antagonistic patients with high Intimacy impairment tend to be more socially withdrawn, which in turn reduces "aggression opportunities." On the contrary, it might be that some antagonistic patients involved in a love relationship-which could indicate some degree of Intimacy functioning-are more prone to IPV since they have a more "direct access" to a victim. This counterintuitive result is somewhat compatible with the DSM-5 AMPD antisocial PD diagnosis*psychopathy specifier interactions that were reported for the prediction of dysfunctional outcomes (Miller et al., 2018); indeed, three out of four of the significant interactions reported had a negative coefficient, one of which being for the statistical prediction of proactive (premeditated, goal-directed) aggression.

Another observation is that the size of the statistically significant interactions reported was higher for the outpatient $(1.1 \%-1.9 \%)$ than the community sample (0.4\%-0.8\%). Personality dysfunction and traits also accounted for more explained variance in the former sample (up to 29\%) than in the latter (17-19\%), when looking at main effects. Aggression is an extremely complex, multidetermined phenomenon (J. J. Allen et al., 2018), and the importance of each factor likely varies depending on the population of reference. It might be that, among personality disordered patients, dysfunction and pathological traits (including their interactions) are more elevated and account for more variance for some outcomes (e.g., aggression), thus supplanting other factors that could otherwise play a more important role in the general population (e.g., neighborhood and socioeconomic status). Indeed, since a categorical PD diagnosis is a risk factor for violence (Yu et al., 2012), it would lend support to the hypothesis that personality and aggression share more variance in PD samples. Nonetheless, in both clinical and nonclinical populations, even small-size effects are important to consider when it comes to aggression, considering its multicausal origin and high societal impact (Hyatt et al., 2020). In an assessment context, in addition to taking into account a patient or inmate's general traits (as suggested by Dunne et al., 2018), the level of personality impairment might add precision to the risk a clinician would attribute to that individual. When placed in the broader AMPD/ ICD-11 literature, the present results support the idea that the field could benefit from a more systematic investigation of interactions.

The two scoring methods globally generated similar interactions, with a slight advantage for Krueger et al.'s algorithms (for which two additional interactions were found in the community sample). This might suggest that the absence of some key facets in APA's method (e.g., Hostility and Risk Taking) makes it a less ideal procedure to study aggression. The size of the interaction terms (i.e., explained variance) was very similar, though. In all cases, the scope of our conclusions is therefore strengthened, given that the presence (or absence) of significant interactions was not simply an artifact of an arguably suboptimal scoring method for the specific application under investigation.

When considering the interaction effects (i.e., the addition of explained variance of significant interactions), it is not clear if a total score versus four-element personality dysfunction structure is optimal (in the case of the AMPD); indeed, both generated statistically significant interaction effects of comparable size. However, it must be noted that, when examining main effects (see Tables 2 and 3), results were less consistent. The Total score was significant for the community sample but not for the outpatients, while both had at least one statistically significant AMPD Criterion A element, suggesting an advantage of the four-factor structure over the singlefactor structure in the latter sample (even with the use of the Predicted $R^{2}$, which prevents overfitting). In addition, as expected, outpatients and community participants with more Empathy impairment were more likely to commit physical aggression; on the other hand, outpatients (but not community participants) with more Identity impairment were less likely to do so.

When considering the trait regression weights, our results make clear that the scoring procedure used and the other predictors inserted into the model have an important impact on the significance level for traits (especially for outpatients), at least for main effects. Disinhibition and Psychoticism (and Antagonism, for community participants) were the most robust and consistent predictors, but Detachment and Negative Affectivity (low) also emerged as significant in at least one model. Taken as a whole, the present results are partly compatible with Dunne et al.'s (2021), Dowgwillo et al.'s (2016), and Sleep et al.'s (2018) results. In the present study, the discrepancies found across models, in terms of statistically significant traits, might shed some light on the inconsistencies of past research, as even small modifications (e.g., the scoring procedure for traits) led to divergent results; thus, additionally changing the population of reference (general population vs. inmates vs. psychiatric patients vs. IPV perpetrators) or the instruments used (e.g., full vs. brief PID-5) will likely affect the associations.

Nonetheless, the present study was the first to highlight the significant role of Psychoticism as a predictor (in both the community and the outpatient samples). Psychoticism might notably capture the transient psychotic symptoms (e.g., paranoid ideation) and/or dissociative symptoms present in some individuals with 
borderline PD (Bach et al., 2020). In addition, Psychoticism was moderated by personality dysfunction (Intimacy, Total score) in the community sample. Of note, interpreting this interaction is complicated considering that the placement of Psychoticism itself within emergent PD models is contentious. In the AMPD, Psychoticism was assigned to Criterion B as a trait, breaking with the psychodynamic tradition of personality organization models (Caligor et al., 2018; Lingiardi \& McWilliams, 2017), in which reality testing impairment represents a structural element, much like identity or interpersonal/object relations, that contributes to determine the level of personality pathology. In the ICD11, however, Psychoticism (or schizotypy) is not included in the PD model, since its constituting features are assigned to the schizophrenia and other primary psychotic disorders category; some psychotic-like symptoms are included in the general PD definition, but it does not correspond to Psychoticism proper, as such symptoms must be associated with situations of high affective arousal (Bach et al., 2020). The present results might fuel further reflection on this topic, for both the AMPD and the ICD-11.

\section{Limitations}

First, even if the study had strong statistical power, especially for the community sample (e.g., effects sizes in the $<1 \%$ range could be detected), it cannot be ruled out that the high number of interaction models tested increased Type I error. Since this was the first study probing interactions in the prediction of aggression, however, a confirmatory approach restricting analyses to a few models or setting a more conservative $\alpha$ level did not appear optimal at this time. As an initial step, a more flexible approach allowing testing for multiple combinations seemed justifiable in the absence of specific hypotheses pertaining to significant interactions or effect sizes. Still, some methodological considerations and results might partially mitigate those concerns, since some interactions were reported in both samples (e.g., Empathy*Disinhibition, Intimacy*Antagonism) and some results in the community sample reached a more conservative significance threshold (i.e., $p<0.01$; e.g., Total score*Antagonism, Empathy*Detachment). The theoretical soundness (e.g., in line with psychodynamic models such as Kernberg's, more personality pathology moderated the effect of traits on aggression [in all but one model]) and overall consistency of results (e.g., the interpersonal component of dysfunction is at the core of all significant interactions, in both samples) also mitigates concerns of Type I error. Second, our results could not generate a very precise causal sequence for two reasons: we used a cross-sectional design and the BPAQ-SF Physical Aggression subscale is a broad and nonspecific measure of perpetrated aggressions (e.g., it does not offer specific information pertaining to frequency or severity of aggression). Finally, the monomethod design (e.g., the absence of informant-report or behavioral data regarding aggression) also limits the scope of our conclusions.

\section{Future directions}

It would be important to identify the interaction of personality dysfunction and specific trait facets (in the case of the AMPD). Since interactions were not studied before, it seemed parsimonious and compatible with the ICD-11 (which does not have facets) to start with domains; future research could narrow down to important facets using more specific hypotheses based on previous research (e.g., Dunne et al., 2018, e.g., Hostility and Risk Taking could be especially predictive in patients with high personality impairment). In addition, it would be relevant to identify if dysfunction*trait interactions can be used as prognostic factors for clinical purposes (e.g., violent, disinhibited patients with high personality impairment could be less responsive to treatment than their low personality impairment counterparts). Finally, identifying interactions through a multimethod design would be highly relevant.

\section{CONCLUSION}

The main result of this study is that some small-size dysfunction*trait interaction effects were found in the statistical prediction of aggression. The results have the potential to be useful and informative to both ICD-11 and AMPD researchers and clinicians. A major strength of this study is the use of two samples, including an appreciably large sample of outpatients with PD, which adds substantially to the robustness and generalizability of conclusions. These results suggest that interaction analyses might further inform, to some degree, about the contentious discussion pertaining to the potential redundancy between dysfunction and traits, the optimal AMPD Criterion A structure, as well as clinical assessment based on AMPD/ICD-11 PD frameworks.

\section{ACKNOWLEDGEMENTS}

This research was supported by research grants from the Social Sciences and Humanities Research Council of Canada to Claudia Savard and Dominick Gamache for a 
project entitled "A computer-based assessment procedure to improve services accessibility and to establish profiles of clients with dysfunctional personality traits" (4A Partnership Engage Grant 2017). It was also financially supported by a sponsorship granted to Claudia Savard, Dominick Gamache, and Marc Tremblay by the Centre intégré universitaire de santé et de services sociaux de la Capitale-Nationale [Integrated University Health and Social Services Center of the CapitaleNationale], Quebec City, Canada. We gratefully thank Renée-Claude Dompierre, Marie-Audrey Lavoie, Evens Villeneuve, and the clinical assessment team at the Centre de traitement le Faubourg Saint-Jean for their invaluable contribution to data collection.

\section{CONFLICT OF INTEREST}

None.

\section{AUTHOR CONTRIBUTION}

PL: study concept and design, statistical analyses, initial draft. CS: data acquisition, obtained funding, review. DDV: review. JF: data acquisition, review. MP: data acquisition, review. ML: data acquisition, review. MT: data acquisition, obtained funding. DG: study concept and design, obtained funding, review.

\section{ETHICS STATEMENT}

The study was ethically approved by the Université du Québec à Trois-Rivières, Université Laval, the Centre intégré universitaire de santé et de services sociaux de la Capitale-Nationale [Integrated University Health and Social Services Center of the Capitale-Nationale] Sectoral Research Ethics Committee in Neurosciences and Mental Health. Procedures were in accordance with the 1964 Helsinki declaration and its subsequent revisions and amendments, or comparable ethical guidelines. Written consent was obtained from every patient prior to the use of their data; provision of clinical services was not contingent upon patients' decision to grant this access.

\section{DATA AVAILABILITY STATEMENT}

The data that support the findings of this study are available on request from the corresponding author. The data are not publicly available due to privacy or ethical restrictions.

\section{ORCID}

Philippe Leclerc (10) https://orcid.org/0000-0003-2609-9275 Claudia Savard (10 https://orcid.org/0000-0002-1286-0257 Dominick Gamache (i) https://orcid.org/0000-0002-8735$712 \mathrm{X}$

\section{ENDNOTE}

${ }^{1}$ The AMPD Criterion B pertains to 25 pathological personality lower-order traits ("facets") organized into five higher-order traits (“domains"; i.e., Negative Affectivity, Detachment, Antagonism, Disinhibition, and Psychoticism); the ICD-11 model does not have lower-order facets (Bach \& Simonsen, 2021). Unless specified otherwise, the word "trait" is used throughout the text to refer to both AMPD domains and ICD-11 traits, for the sake of simplicity. For a thorough description of the similarities and differences between the AMPD and the ICD-11 PD model, see, for example, Bach and First (2018) and Tyrer et al. (2019).

\section{REFERENCES}

Allen, D. M. (1974). The relationship between variable selection and data augmentation and a method for prediction. Technometrics, 16, 125-127. https://doi.org/10.1080/00401706. 1974.10489157

Allen, J. J., Anderson, C. A., \& Bushman, B. J. (2018). The general aggression model. Current Opinion in Psychology, 19, 75-80. https://doi.org/10.1016/j.copsyc.2017.03.034

American Psychiatric Association. (2013). Diagnostic and statistical manual of mental disorders (5th ed.) DSM-5. American Psychiatric Publishing.

Bach, B., \& First, M. B. (2018). Application of the ICD-11 classification of personality disorders. BMC Psychiatry, 18, 351.

Bach, B., \& Simonsen, S. (2021). How does level of personality functioning inform clinical management and treatment? Implications for ICD-11 classification of personality disorder severity. Current Opinion in Psychiatry, 34, 54-63. https://doi.org/10. 1097/YCO.0000000000000658

Bach, B., Kerber, A., Aluja, A., Bastiaens, T., Keeley, J. W., Claes, L., Fossati, A., Gutierrez, F., Oliveira, S. E. S., Pires, R., Riegel, K. D., Rolland, J. P., Roskam, I., Sellbom, M., Somma, A., Spanemberg, L., Strus, W., Thimm, J. C., Wright, A. G. C., \& Zimmermann, J. (2020). International assessment of DSM-5 and ICD-11 personality disorder traits: Toward a common nosology in DSM-5.1. Psychopathology, 53, 179-188. https://doi.org/10.1159/000507589

Benzi, I. M. A., Preti, E., Di Pierro, R., Clarkin, J. F., \& Madeddu, F. (2019). Maladaptive personality traits and psychological distress in adolescence: The moderating role of personality functioning. Personality and Individual Differences, 140, 33-40. https://doi. org/10.1016/j.paid.2018.06.026

Bryant, F. B., \& Smith, B. D. (2001). Refining the architecture of aggression: A measurement model for the Buss-Perry aggression questionnaire. Journal of Research in Personality, 35, 138-167. https://doi.org/10.1006/jrpe.2000.2302

Buer Christensen, T., Eikenaes, I., Hummelen, B., Pedersen, G., Nysæter, T.-E., Bender, D. S., Skodol, A. E., \& Selvik, S. G. (2020). Level of personality functioning as a predictor of psychosocial functioning-Concurrent validity of criterion A. Personality Disorders: Theory, Research, and Treatment, 11, 79-90. https://doi.org/10.1037/per0000352

Buss, A. H., \& Perry, M. (1992). The aggression questionnaire. Journal of Personality and Social Psychology, 63, 452-459. https:// doi.org/10.1037/0022-3514.63.3.452 
Caligor, E., Kernberg, O. F., Clarkin, J. F., \& Yeomans, F. E. (2018). Psychodynamic therapy for personality pathology: Treating self and interpersonal functioning. American Psychiatric Publishing.

Darlington, R. B., \& Hayes, A. F. (2016). Regression analysis and linear models: Concepts, applications, and implementation. Guilford.

Dowgwillo, E. A., Ménard, K. S., Krueger, R. F., \& Pincus, A. L. (2016). DSM-5 pathological personality traits and intimate partner violence among male and female college students. Violence and Victims, 31, 416-437. https://doi.org/10.1891/0886-6708. VV-D-14-00109

Dunne, A. L., Gilbert, F., \& Daffern, M. (2018). Investigating the relationship between DSM-5 personality disorder domains and facets and aggression in an offender population using the personality inventory for the DSM-5. Journal of Personality Disorders, 32, 668-693. https://doi.org/10.1521/pedi_2017_31_322

Dunne, A., Trounson, J., Skues, J., Pfeifer, J., Ogloff, J., \& Daffern, M. (2021). The personality inventory for DSM-5-brief form: An examination of internal consistency, factor structure, and relationship to aggression in an incarcerated offender sample. Assessment, 28, 1136-1146. https://doi.org/10.1177/ 1073191120916790

Durand, C. P. (2013). Does raising type 1 error rate improve power to detect interactions in linear regression models? A simulation study. PLoS ONE, 8, e71079. https://doi.org/10.1371/journal. pone.0071079

Gamache, D., Savard, C., Leclerc, P., \& Côté, A. (2019). Introducing a short self-report for the assessment of DSM-5 level of personality functioning for personality disorders: The self and interpersonal functioning scale. Personality Disorders: Theory, Research, and Treatment, 10, 438-447. https://doi.org/10.1037/ per0000335

Gamache, D., Savard, C., Lemieux, R., \& Berthelot, N. (2021) Impact of level of personality pathology on affective, behavioral, and thought problems in pregnant women during the coronavirus disease 2019 pandemic. Personality Disorders: Theory, Research, and Treatment. advance online publication. https://doi.org/10.1037/per0000479

Gamache, D., Savard, C., Leclerc, P., Payant, M., Berthelot, N., Côté, A., Faucher, J., Lampron, M., Lemieux, R., Mayrand, K., Nolin, M.-C., \& Tremblay, M. (2021). A proposed classification of ICD-11 severity degrees of personality pathology using the self and interpersonal functioning scale. Frontiers in Psychiatry, 12, 292. https://doi.org/10.3389/fpsyt.2021.628057

Genoud, P., \& Zimmermann, G. (2009). French version of the 12-item aggression questionnaire: Preliminary Psychometric Properties. https://www3.unifr.ch/cerf/fr/assets/public/pdf/ pages\%20personnelles/Genoud\%20Philippe/2009_SSP.pdf

Hopwood, C. J., Kotov, R., Krueger, R. F., Watson, D., Widiger, T. A., Althoff, R. R., Ansell, E. B., Bach, B., Bagby, R. M., Blais, M. A., Bornovalova, M. A., Chmielewski, M., Cicero, D. C., Conway, C., De Clercq, B., De Fruyt, F., Docherty, A. R., Eaton, N. R., Edens, J. F., \& Zimmermann, J. (2018). The time has come for dimensional personality disorder diagnosis. Personality and Mental Health, 12, 82-86. https://doi.org/10.1002/pmh.1408

Hummelen, B., Braeken, J., Buer Christensen, T., Nysaeter, T. E., Germans Selvik, S., Walther, K., et al. (2020). A psychometric analysis of the structured clinical interview for the DSM-5 alternative model for personality disorders module I (SCID5-AMPD-I): Level of personality functioning scale. Assessment. advance online publication, 28, 1320-1333. https://doi.org/10. $1177 / 1073191120967972$

Hyatt, C. S., Sleep, C. E., Weiss, B. M., \& Miller, J. D. (2018). Personality and aggression: A general trait perspective. In A. T. Vazsonyi, D. J. Flannery, \& M. DeLisi (Eds.), The Cambridge handbook of violent behavior and aggression (2nd ed.) (pp. 221-238). Cambridge University Press.

Hyatt, C. S., Chester, D. S., Zeichner, A., \& Miller, J. D. (2020). Facet-level analysis of the relations between personality and laboratory aggression. Aggressive Behavior, 46, 266-277. https:// doi.org/10.1002/ab.21887

Iacobucci, D., Schneider, M. J., Popovich, D. L., \& Bakamitsos, G. A. (2016). Mean centering helps alleviate "micro" but not "macro" multicollinearity. Behavior Research Methods, 48, 1308-1317. https://doi.org/10.3758/s13428-0150624-x

Krueger, R. F., Derringer, J., Markon, K. E., Watson, D., \& Skodol, A. E. (2012). Initial construction of a maladaptive personality trait model and inventory for DSM-5. Psychological Medicine, 42, 1879-1890. https://doi.org/10.1017/ S0033291711002674

Lingiardi, V., \& McWilliams, N. (2017). Psychodynamic diagnostic manual: PDM-2 (2nd ed.). Guilford.

Maples, J. L., Carter, N. T., Few, L. R., Crego, C., Gore, W. L., Samuel, D. B., Williamson, R. L., Lynam, D. R., Widiger, T. A., Markon, K. E., Krueger, R. F., \& Miller, J. D. (2015). Testing whether the DSM-5 personality disorder trait model can be measured with a reduced set of items: An item response theory investigation of the personality inventory for DSM-5. Psychological Assessment, 27, 1195-1210. https://doi.org/10.1037/ pas0000120

McCabe, G. A., \& Widiger, T. A. (2020). A comprehensive comparison of the ICD-11 and DSM-5 section III personality disorder models. Psychological Assessment, 32, 72-84. https://doi.org/10. 1037/pas0000772

Meehan, K. B., Siefert, C., Sexton, J., \& Huprich, S. K. (2019). Expanding the role of levels of personality functioning in personality disorder taxonomy: Commentary on "Criterion A of the AMPD in HiTOP". Journal of Personality Assessment, 101, 367-373. https://doi.org/10.1080/00223891.2018.1551228

Miller, J. D., Lamkin, J., Maples-Keller, J. L., Sleep, C. E., \& Lynam, D. R. (2018). A test of the empirical profile and coherence of the DSM-5 psychopathy specifier. Psychological Assessment, 30, 870-881. https://doi.org/10.1037/pas0000536

Morey, L. C. (2017). Development and initial evaluation of a selfreport form of the DSM-5 level of personality functioning scale. Psychological Assessment, 29, 1302-1308. https://doi.org/10. 1037/pas0000450

Morey, L. C. (2019). Thoughts on the assessment of the DSM-5 alternative model for personality disorders: Comment on Sleep et al. (2019). Psychological Assessment, 31, 1192-1199. https:// doi.org/10.1037/pas0000710

Mundfrom, D. J., Perrett, D., Schaffer, J., Piccone, A., \& Roozeboom, M. (2006). Bonferroni adjustments in tests for regression coefficients. Multiple Linear Regression Viewpoints, 32, 1-6.

Munro, O. E., \& Sellbom, M. (2020). Elucidating the relationship between borderline personality disorder and intimate partner 
violence. Personality and Mental Health, 14, 284-303. https:// doi.org/10.1002/pmh.1480

Roskam, I., Galdiolo, S., Hansenne, M., Massoudi, K., Rossier, J., Gicquel, L., \& Rolland, J.-P. (2015). The psychometric properties of the French version of the personality inventory for DSM5. PLoS ONE, 10, e0133413. https://doi.org/10.1371/journal. pone. 0133413

Sexton, J., Hilton, M., Benson, S., \& Rosen, A. (2019). Exploring Kernberg's model of personality functioning as a moderator of traits: Focus on DSM-5's section III alternative model of personality disorder. Journal of the American Psychoanalytic Association, 67, 1047-1055. https://doi.org/10.1177/0003065119898772

Siefert, C. J., Sexton, J., Meehan, K., Nelson, S., Haggerty, G., Dauphin, B., \& Huprich, S. (2020). Development of a short form for the DSM-5 levels of personality functioning questionnaire. Journal of Personality Assessment, 102, 1-11. https://doi. org/10.1080/00223891.2019.1594842

Sleep, C. E., Hyatt, C. S., Lamkin, J., Maples-Keller, J. L., \& Miller, J. D. (2018). Examining the relations among the DSM-5 alternative model of personality, the five-factor model, and externalizing and internalizing behavior. Personality Disorders: Theory, Research, and Treatment, 9, 379-384. https://doi.org/10. 1037/per0000240

Sleep, C. E., Lynam, D. R., Widiger, T. A., Crowe, M. L., \& Miller, J. D. (2019). Difficulties with the conceptualization and assessment of criterion A in the DSM-5 alternative model of personality disorder: A reply to Morey (2019). Psychological Assessment, 31, 1200-1205. https://doi.org/10.1037/pas0000758

Tyrer, P., Mulder, R., Kim, Y. R., \& Crawford, M. J. (2019). The development of the ICD-11 classification of personality disorders: An amalgam of science, pragmatism, and politics. Annual Review of Clinical Psychology, 15, 481-502. https://doi.org/10. 1146/annurev-clinpsy-050718-095736

Verheul, R., Andrea, H., Berghout, C. C., Dolan, C., Busschbach, J. J. V., van der Kroft, P. J. A., Bateman, A. W., \& Fonagy, P. (2008). Severity indices of personality problems (SIPP-118): Development, factor structure, reliability, and validity. Psychological Assessment, 20, 23-34. https://doi.org/10. 1037/1040-3590.20.1.23

Watters, C. A., Sellbom, M., \& Bagby, R. M. (2019). Comparing two domain scoring methods for the personality inventory for DSM-5. Psychological Assessment, 31, 1125-1134. https://doi. org/10.1037/pas0000739
Waugh, M. H., McClain, C. M., Mariotti, E. C., Mulay, A. L., DeVore, E. N., Lenger, K. A., Russell, A. N., Florimbio, A. R., Lewis, K. C., Ridenour, J. M., \& Beevers, L. G. (2021). Comparative content analysis of self-report scales for level of personality functioning. Journal of Personality Assessment, 103, 161-173. https://doi.org/10.1080/00223891.2019.1705464

Weekers, L. C., Hutsebaut, J., \& Kamphuis, J. H. (2019). The level of personality functioning scale-brief form 2.0: Update of a brief instrument for assessing level of personality functioning. Personality and Mental Health, 13, 3-14. https://doi.org/10.1002/ pmh.1434

Yu, R., Geddes, J. R., \& Fazel, S. (2012). Personality disorders, violence, and antisocial behavior: A systematic review and meta-regression analysis. Journal of Personality Disorders, 26, 775-792. https://doi.org/10.1521/pedi.2012.26.5.775

Zimmermann, J., Kerber, A., Rek, K., Hopwood, C. J., \& Krueger, R. F. (2019). A brief but comprehensive review of research on the alternative DSM-5 model for personality disorders. Current Psychiatry Reports, 21, 92. https://doi.org/10.1007/ s11920-019-1079-z

Zimmermann, J., Müller, S., Bach, B., Hutsebaut, J., Hummelen, B., \& Fischer, F. (2020). A common metric for selfreported severity of personality disorder. Psychopathology, 53, 168-178. https://doi.org/10.1159/000507377

\section{SUPPORTING INFORMATION}

Additional supporting information may be found online in the Supporting Information section at the end of this article.

How to cite this article: Leclerc, P., Savard, C., Vachon, D. D., Faucher, J., Payant, M., Lampron, M., Tremblay, M., \& Gamache, D. (2021). Analysis of the interaction between personality dysfunction and traits in the statistical prediction of physical aggression: Results from outpatient and community samples. Personality and Mental Health, 1-14. https://doi.org/10.1002/pmh.1522 